STRUCTURE REPORTS

ISSN 1600-5368

Received 20 October 2014

Accepted 3 November 2014

Edited by L. Farrugia, University of Glasgow, Scotland

Keywords: crystal structure; gadolinium; rhenium; silicon; intermetallic compound; ternary silicide

CCDC reference: 1032373

Supporting information: this article has

supporting information at journals.iucr.org/e

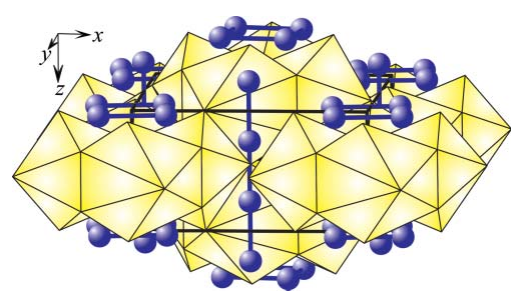

OPEN $\odot$ ACCESS

\section{Crystal structure of the ternary silicide $G_{d_{2}} \operatorname{Re}_{3} S_{5}$}

\author{
Vitaliia Fedyna, ${ }^{\mathrm{a} *}$ Roksolana Kozak ${ }^{\mathrm{b}}$ and Roman Gladyshevskii ${ }^{\mathrm{a}}$
}

${ }^{\text {a}}$ Department of Inorganic Chemistry, Ivan Franko National University of Lviv, Kyryla i Mefodiya st. 6, UA-79005 Lviv,
Ukraine, and ${ }^{\mathbf{b}}$ Laboratory of Crystallography, Department of Materials, ETH Zurich, Vladimir-Prelog-Weg 5, CH-8093
Zurich, Switzerland. *Correspondence e-mail: vitaliia.fedyna@gmail.com

A single crystal of the title compound, the ternary silicide digadolinium trirhenium pentasilicide, $\mathrm{Gd}_{2} \mathrm{Re}_{3} \mathrm{Si}_{5}$, was isolated from an alloy of nominal composition $\mathrm{Gd}_{20} \mathrm{Re}_{30} \mathrm{Si}_{50}$ synthesized by arc melting and investigated by $\mathrm{X}$-ray single-crystal diffraction. Its crystal structure belongs to the $\mathrm{U}_{2} \mathrm{Mn}_{3} \mathrm{Si}_{5}$ structure type. All atoms in the asymmetric lie on special positions. The Gd site has site symmetry $m$. .; the two $\mathrm{Mn}$ atoms have site symmetries $m$.. and 2.22; the three $\mathrm{Si}$ atoms have site symmetries $m . ., . .2$ and 4 .. . The coordination polyhedra of the Gd atoms have 21 vertices, while those of the Re atoms are cubooctahedra and 13-vertex polyhedra. The $\mathrm{Si}$ atoms are arranged as tricapped trigonal prisms, bicapped square antiprisms, or 11-vertex polyhedra. The crystal structure of the title compound is also related to the structure types $\mathrm{CaBe}_{2} \mathrm{Ge}_{2}$ and $\mathrm{W}_{5} \mathrm{Si}_{3}$. It can be represented as a stacking of Gd-centred polyhedra of composition [ $\mathrm{GdSi}_{9}$ ]. The Re atoms form infinite chains with an $\mathrm{Re}-\mathrm{Re}$ distance of 2.78163 (5) $\AA$ and isolated squares with an $\mathrm{Re}-\mathrm{Re}$ distance of 2.9683 (6) $\AA$.

\section{Chemical context}

Four structure types of composition $R_{2} T_{3} \mathrm{Si}_{5}$ are known for the systems $R-T$-Si $(R=$ rare-earth element, $T=d$-block element): $\mathrm{U}_{2} \mathrm{Mn}_{3} \mathrm{Si}_{5}$ (Yarmolyuk et al., 1977) (Pearson symbol tP40, space group P4/mnc), $\mathrm{U}_{2} \mathrm{Co}_{3} \mathrm{Si}_{5}$ (Akselrud et al., 1977) (oI40, Ibam), $\mathrm{Nd}_{2} \mathrm{Os}_{3} \mathrm{Si}_{5}$ (Rizzoli et al., 2004) (tP48, P4/mnc) and $\mathrm{Lu}_{2} \mathrm{Co}_{3} \mathrm{Si}_{5}$ (Chabot \& Parthé, 1985) $(m S 40, C 2 / c)$. The structure type $\mathrm{U}_{2} \mathrm{Mn}_{3} \mathrm{Si}_{5}$ has representatives in the systems $R-\mathrm{Mn}-\mathrm{Si}(R=\mathrm{Y}, \mathrm{Gd}-\mathrm{Lu}), R-\mathrm{Re}-\mathrm{Si}(R=\mathrm{Y}, \mathrm{La}-\mathrm{Nd}, \mathrm{Sm}, \mathrm{Gd}-$ $\mathrm{Tm}), R-\mathrm{Fe}-\mathrm{Si}(R=\mathrm{Sc}, \mathrm{Y}, \mathrm{Sm}, \mathrm{Gd}-\mathrm{Lu}), R-\mathrm{Ru}-\mathrm{Si}(R=\mathrm{Sm}, \mathrm{Er}$, $\mathrm{Lu}$ ), whereas the structure type $\mathrm{U}_{2} \mathrm{Co}_{3} \mathrm{Si}_{5}$ has been found in the systems $R-\mathrm{Ru}-\mathrm{Si}(R=\mathrm{Tb}, \mathrm{Er}), R-\mathrm{Co}-\mathrm{Si}(R=\mathrm{Sc}, \mathrm{Y}, \mathrm{Ce}$, Gd-Er), $R-\mathrm{Rh}-\mathrm{Si}(R=\mathrm{Y}, \mathrm{La}, \mathrm{Ce}, \mathrm{Nd}, \mathrm{Sm}, \mathrm{Gd}-\mathrm{Er}), R-\mathrm{Ir}-\mathrm{Si}$ $(R=\mathrm{Y}, \mathrm{Ce}, \mathrm{Tb}, \mathrm{Lu}), R-\mathrm{Ni}-\mathrm{Si}(R=\mathrm{Y}, \mathrm{Ce}, \mathrm{Nd}, \mathrm{Sm}, \mathrm{Gd}-\mathrm{Tm})$, $R-\mathrm{Pt}-\mathrm{Si}(R=\mathrm{Ce}, \mathrm{Sm})$, and $R-\mathrm{Pd}-\mathrm{Si}(R=\mathrm{Ce}, \mathrm{Sm})$, the structure type $\mathrm{Nd}_{2} \mathrm{Os}_{3} \mathrm{Si}_{5}$ in the systems $R-\mathrm{Os}-\mathrm{Si}(R=\mathrm{Nd}, \mathrm{Eu})$, and the structure type $\mathrm{Lu}_{2} \mathrm{Co}_{3} \mathrm{Si}_{5}$ in the systems $R-\mathrm{Co}-\mathrm{Si}(R=\mathrm{Y}$, $\mathrm{Tb}, \mathrm{Dy}, \mathrm{Lu}), R-\mathrm{Rh}-\mathrm{Si}(R=\mathrm{Y}, \mathrm{Tb}, \mathrm{Dy})$ and $R-\mathrm{Ni}-\mathrm{Si}(R=\mathrm{Lu})$ (Villars \& Cenzual, 2013).

\section{Structural commentary}

The existence of the compound $\mathrm{Gd}_{2} \mathrm{Re}_{3} \mathrm{Si}_{5}$ has been reported earlier (Bodak et al., 1978). The unit-cell parameters were determined and the structure type was assigned. A complete investigation of the crystal structure by X-ray single crystal diffraction has now been undertaken. The coordination polyhedra of the $\mathrm{Gd}$ atoms have 21 vertexes, whereas those of the Re atoms are cubooctahedra or 13-vertex polyhedra, and the Si atoms tricapped trigonal prisms, bicapped square anti- 


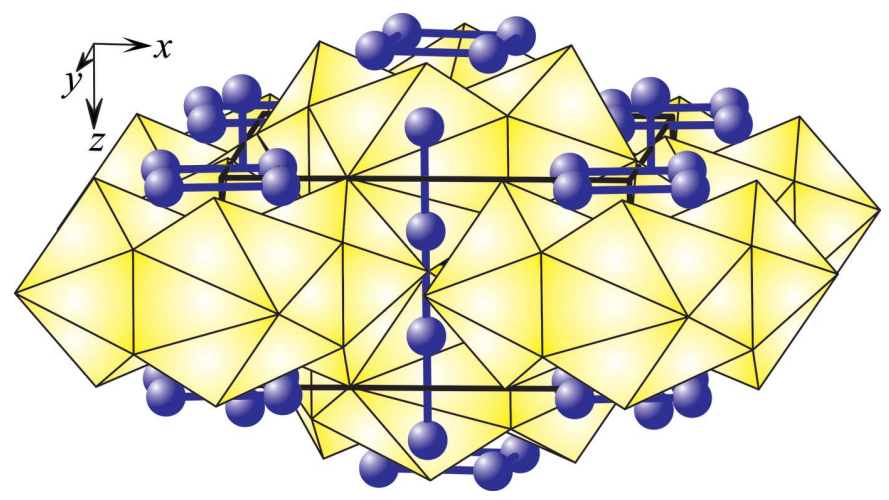

Figure 1

Stacking of Gd-centred polyhedra in the structure of the compound $\mathrm{Gd}_{2} \mathrm{Re}_{3} \mathrm{Si}_{5}$ with displacement ellipsoids drawn at the $99 \%$ probability level.

prisms, or 11-vertex polyhedra. The $\mathrm{U}_{2} \mathrm{Mn}_{3} \mathrm{Si}_{5}$-type structure is closely related to the structure type $\mathrm{BaAl}_{4}$ and its ordered derivative $\mathrm{CaBe}_{2} \mathrm{Ge}_{2}$. In particular, the $\mathrm{U}_{2} \mathrm{Mn}_{3} \mathrm{Si}_{5}$-type can be considered to be formed by one-dimensional structural fragments of the structure type $\mathrm{CaBe}_{2} \mathrm{Ge}_{2}$, running parallel to the direction [001]. There also exists a relationship between the structure types $\mathrm{U}_{2} \mathrm{Mn}_{3} \mathrm{Si}_{5}$ and $\mathrm{W}_{5} \mathrm{Si}_{3}$. Fragments which can be viewed as deformed square antiprisms are common to both structures. The crystal structure of $\mathrm{Gd}_{2} \mathrm{Re}_{3} \mathrm{Si}_{5}$ can also be represented as a stacking of Gd-centred polyhedra of composition $\left[\mathrm{GdSi}_{9}\right.$ ], located at $z=0$ and $\frac{1}{2}$ (Fig. 1) (Parthé et al., 1993). The $\mathrm{Re}$ atoms form infinite chains with an $\mathrm{Re}-\mathrm{Re}$ distance of 2.78163 (5) $\AA$ and isolated squares with an $\mathrm{Re}-\mathrm{Re}$ distance of 2.9683 (6) $\AA$.

\section{Synthesis and crystallization}

An alloy of nominal atom percent composition $\operatorname{Gd}_{20} \mathrm{Re}_{30} \mathrm{Si}_{50}$ was synthesized from the high-purity elements by arc melting on a water-cooled copper plate under a purified argon atmosphere, using titanium as a getter and a tungsten electrode. The weight loss during the sample preparation was less than $0.5 \%$ of the total mass $(1 \mathrm{~g})$. The alloy was placed into an $\mathrm{Al}_{2} \mathrm{O}_{3}$ crucible and inserted into a tantalum container, which was then sealed by welding, leaving the sample under an argon atmosphere. The sample, wrapped in tantalum foil, was heated to $1623 \mathrm{~K}$ in a muffle furnace at a rate of $200 \mathrm{~K} \mathrm{~h}^{-1}$, held at this temperature for $5 \mathrm{~h}$ and then cooled to room temperature at a rate of $50 \mathrm{~K} \mathrm{~h}^{-1}$.

\section{Refinement details}

A single crystal of well-defined shape was separated from the sample. The structure was solved by direct methods. The

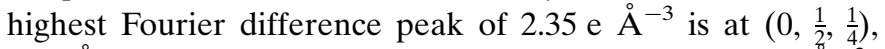

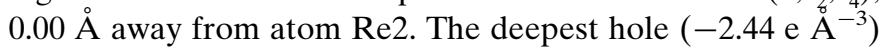
is at $(0.6045,0.3985,0), 1.52 \AA$ away from the Gd atom. Details of the crystal parameters, data collection and the structure refinement details are summarized in Table 1.

Table 1

Experimental details.

Crystal data

Chemical formula

$M_{\text {r }}$

Crystal system, space group

Temperature (K)

$a, c(\AA)$

$V\left(\AA^{3}\right)$

Z

Radiation type

$\mu\left(\mathrm{mm}^{-1}\right)$

Crystal size (mm)

Data collection

Diffractometer

Absorption correction

$T_{\min }, T_{\max }$

No. of measured, independent and observed $[I>2 \sigma(I)]$ reflections

$R_{\text {int }}$

$(\sin \theta / \lambda)_{\max }\left(\AA^{-1}\right)$

Refinement

$R\left[F^{2}>2 \sigma\left(F^{2}\right)\right], w R\left(F^{2}\right), S$

No. of reflections

No. of parameters

$\Delta \rho_{\max }, \Delta \rho_{\min }\left(\mathrm{e} \AA^{-3}\right)$

Computer programs: CrysAlis PRO (Agilent, 2012), SHELXS97 and SHELXL97 (Sheldrick, 2008), WinGX (Farrugia, 2012), DIAMOND (Brandenburg, 2006) and publCIF (Westrip, 2010).

\section{Acknowledgements}

This work was supported by the Ministry of Education and Science of Ukraine under grant No 0112U001279.

\section{References}

Agilent (2012). CrysAlis PRO and CrysAlis RED. Agilent Technologies, Yarnton, England.

Akselrud, L. G., Yarmolyuk, Y. P. \& Gladyshevskii, E. I. (1977). Sov. Phys. Crystallogr. 22, 492-493.

Bodak, O. I., Pecharskii, V. K. \& Gladyshevskii, E. I. (1978). Izv. Akad. Nauk SSSR Neorg. Mater. 14, 251-255.

Brandenburg, K. (2006). DIAMOND. Crystal Impact GbR, Bonn, Germany.

Chabot, B. \& Parthé, E. (1985). J. Less-Common Met. 106, 53-59.

Clark, R. C. \& Reid, J. S. (1995). Acta Cryst. A51, 887-897.

Farrugia, L. J. (2012). J. Appl. Cryst. 45, 849-854.

Parthé, E., Gelato, L., Chabot, B., Penzo, M., Cenzual, K. \& Gladyshevskii, R. (1993). TYPIX. Standardized Data and Crystal Chemical Characterization of Inorganic Structure Types. Heidelberg: Springer-Verlag.

Rizzoli, C., Salamakha, P. S., Sologub, O. L., Belletti, D., Goncalves, A. P. \& Almeida, M. (2004). J. Alloys Compd, 363, 217-222.

Sheldrick, G. M. (2008). Acta Cryst. A64, 112-122.

Villars, P. \& Cenzual, K. (2013). Pearson's Crystal Data. Crystal Structure Database for Inorganic Compounds. Release 2013/14. Materials Park, Ohio: ASM International.

Westrip, S. P. (2010). J. Appl. Cryst. 43, 920-925.

Yarmolyuk, Y. P., Akselrud, L. G. \& Gladyshevskii, E. I. (1977). Sov. Phys. Crystallogr. 22, 358-359. 


\section{supporting information}

Acta Cryst. (2014). E70, 469-470 [doi:10.1107/S1600536814024234]

\section{Crystal structure of the ternary silicide $\mathrm{Gd}_{2} \mathrm{Re}_{3} \mathrm{Si}_{5}$}

\section{Vitaliia Fedyna, Roksolana Kozak and Roman Gladyshevskii}

\section{Computing details}

Data collection: CrysAlis PRO (Agilent, 2012); cell refinement: CrysAlis PRO (Agilent, 2012); data reduction: CrysAlis PRO (Agilent, 2012); program(s) used to solve structure: SHELXS97 (Sheldrick, 2008); program(s) used to refine structure: SHELXL97 (Sheldrick, 2008) and WinGX (Farrugia, 2012); molecular graphics: DIAMOND (Brandenburg, 2006); software used to prepare material for publication: publCIF (Westrip, 2010).

\section{Digadolinium trirhenium pentasilicide}

Crystal data

$\mathrm{Gd}_{2} \mathrm{Re}_{3} \mathrm{Si}_{5}$

$M_{r}=1013.55$

Tetragonal, $P 4 / m n c$

Hall symbol: -P $42 \mathrm{n}$

$a=10.95564$ (13) $\AA$

$c=5.56326(11) \AA$

$V=667.74(2) \AA^{3}$

$Z=4$

$F(000)=1692$

$D_{\mathrm{x}}=10.082 \mathrm{Mg} \mathrm{m}^{-3}$

Mo $K \alpha$ radiation, $\lambda=0.71073 \AA$

Cell parameters from 8564 reflections

$\theta=1.9-29.4^{\circ}$

$\mu=74.55 \mathrm{~mm}^{-1}$

$T=293 \mathrm{~K}$

Irregular, grey

$0.16 \times 0.10 \times 0.02 \mathrm{~mm}$

\section{Data collection}

Agilent Xcalibur Onyx diffractometer

Radiation source: Enhance (Mo) X-ray Source

Graphite monochromator

CCD scans

Absorption correction: analytical [CrysAlis PRO (Agilent, 2012; analytical numeric absorption correction using a multifaceted crystal model (Clark \& Reid, 1995)]

$T_{\min }=0.015, T_{\max }=0.194$

11378 measured reflections

502 independent reflections

481 reflections with $I>2 \sigma(I)$

$R_{\text {int }}=0.062$

$\theta_{\text {max }}=29.5^{\circ}, \theta_{\min }=2.6^{\circ}$

$h=-14 \rightarrow 14$

$k=-15 \rightarrow 14$

$l=-7 \rightarrow 7$

\section{Refinement}

Refinement on $F^{2}$

Least-squares matrix: full

$R\left[F^{2}>2 \sigma\left(F^{2}\right)\right]=0.025$

$w R\left(F^{2}\right)=0.063$

$S=1.17$

502 reflections

31 parameters

0 restraints

Primary atom site location: structure-invariant direct methods

Secondary atom site location: difference Fourier

$$
\text { map }
$$

$w=1 /\left[\sigma^{2}\left(F_{0}^{2}\right)+(0.0342 P)^{2}+8.350 P\right]$

where $P=\left(F_{\mathrm{o}}{ }^{2}+2 F_{\mathrm{c}}{ }^{2}\right) / 3$

$(\Delta / \sigma)_{\max }<0.001$

$\Delta \rho_{\max }=2.35$ e $\AA^{-3}$

$\Delta \rho_{\min }=-2.44$ e $\AA^{-3}$

Extinction correction: SHELXL97 (Sheldrick, 2008), $\mathrm{Fc}^{*}=\mathrm{kFc}\left[1+0.001 \mathrm{xFc}^{2} \lambda^{3} / \sin (2 \theta)\right]^{-1 / 4}$

Extinction coefficient: 0.00016 (8) 


\section{Special details}

Experimental. Analytical numeric absorption correction using a multifaceted crystal model based on expressions derived by Clark \& Reid (1995).

Geometry. All e.s.d.'s (except the e.s.d. in the dihedral angle between two 1.s. planes) are estimated using the full covariance matrix. The cell e.s.d.'s are taken into account individually in the estimation of e.s.d.'s in distances, angles and torsion angles; correlations between e.s.d.'s in cell parameters are only used when they are defined by crystal symmetry. An approximate (isotropic) treatment of cell e.s.d.'s is used for estimating e.s.d.'s involving 1.s. planes.

Refinement. Refinement of $F^{2}$ against ALL reflections. The weighted $R$-factor $w R$ and goodness of fit $S$ are based on $F^{2}$, conventional $R$-factors $R$ are based on $F$, with $F$ set to zero for negative $F^{2}$. The threshold expression of $F^{2}>\sigma\left(F^{2}\right)$ is used only for calculating $R$-factors(gt) etc. and is not relevant to the choice of reflections for refinement. $R$-factors based on $F^{2}$ are statistically about twice as large as those based on $F$, and $R$ - factors based on ALL data will be even larger.

Fractional atomic coordinates and isotropic or equivalent isotropic displacement parameters $\left(\AA^{2}\right)$

\begin{tabular}{lllll}
\hline & $x$ & $y$ & $z$ & $U_{\text {iso }} * / U_{\text {eq }}$ \\
\hline Gd & $0.26249(5)$ & $0.42271(5)$ & 0.0000 & $0.0187(2)$ \\
Re1 & $0.14676(4)$ & $0.12315(4)$ & 0.0000 & $0.01716(18)$ \\
Re2 & 0.0000 & 0.5000 & 0.2500 & $0.0173(2)$ \\
Si1 & $0.0267(3)$ & $0.3149(3)$ & 0.0000 & $0.0192(6)$ \\
Si2 & $0.17183(18)$ & $0.67183(18)$ & 0.2500 & $0.0145(6)$ \\
Si3 & 0.0000 & 0.0000 & $0.2567(9)$ & $0.0197(9)$ \\
\hline
\end{tabular}

Atomic displacement parameters $\left(\AA^{2}\right)$

\begin{tabular}{lllllll}
\hline & $U^{11}$ & $U^{22}$ & $U^{33}$ & $U^{12}$ & $U^{13}$ & $U^{23}$ \\
\hline Gd & $0.0183(3)$ & $0.0199(3)$ & $0.0179(3)$ & $0.0004(2)$ & 0.000 & 0.000 \\
Re1 & $0.0166(3)$ & $0.0172(3)$ & $0.0177(3)$ & $-0.00060(16)$ & 0.000 & 0.000 \\
Re2 & $0.0169(2)$ & $0.0169(2)$ & $0.0180(3)$ & $0.0003(2)$ & 0.000 & 0.000 \\
Si1 & $0.0207(15)$ & $0.0184(15)$ & $0.0184(14)$ & $0.0029(12)$ & 0.000 & 0.000 \\
Si2 & $0.0130(8)$ & $0.0130(8)$ & $0.0174(13)$ & $0.0005(10)$ & $-0.0025(8)$ & $0.0025(8)$ \\
Si3 & $0.0190(13)$ & $0.0190(13)$ & $0.021(2)$ & 0.000 & 0.000 & 0.000 \\
\hline
\end{tabular}

Geometric parameters $\left(\hat{A},{ }^{\circ}\right)$

\begin{tabular}{|c|c|c|c|}
\hline $\mathrm{Gd}-\mathrm{Si} 1$ & $2.841(4)$ & $\operatorname{Re} 2-\operatorname{Re} 2^{x i v}$ & $2.7816(1)$ \\
\hline $\mathrm{Gd}-\mathrm{Si}^{\mathrm{i}}$ & $2.9604(12)$ & $\operatorname{Re} 2-\operatorname{Re} 2^{\text {viii }}$ & $2.7816(1)$ \\
\hline $\mathrm{Gd}-\mathrm{Si} 1^{\mathrm{ii}}$ & $2.9604(12)$ & $\operatorname{Re} 2-\mathrm{Gd}^{\mathrm{viii}}$ & $3.3047(5)$ \\
\hline $\mathrm{Gd}-\mathrm{Si} 3^{\mathrm{iii}}$ & $3.053(2)$ & $\operatorname{Re} 2-\mathrm{Gd}^{\mathrm{xii}}$ & $3.3047(5)$ \\
\hline $\mathrm{Gd}-\mathrm{Si}^{\mathrm{iv}}$ & $3.053(2)$ & $\operatorname{Re} 2-\mathrm{Gd}^{\mathrm{ii}}$ & $3.3047(5)$ \\
\hline $\mathrm{Gd}-\mathrm{Re} 1^{\mathrm{ii}}$ & $3.1442(3)$ & $\operatorname{Si} 1-\operatorname{Re} 1^{x i}$ & $2.467(4)$ \\
\hline $\mathrm{Gd}-\mathrm{Re} 1^{\mathrm{i}}$ & $3.1442(3)$ & $\operatorname{Si} 1-\operatorname{Re} 2^{\text {viii }}$ & $2.476(3)$ \\
\hline $\mathrm{Gd}-\mathrm{Si} 2^{\mathrm{v}}$ & $3.163(2)$ & $\mathrm{Si} 1-\mathrm{Si} 2^{\mathrm{viii}}$ & $2.585(3)$ \\
\hline $\mathrm{Gd}-\mathrm{Si}^{\mathrm{vi}}$ & $3.163(2)$ & $\mathrm{Si} 1-\mathrm{Si} 2^{\mathrm{xiii}}$ & 2.585 \\
\hline $\mathrm{Gd}-\mathrm{Si} 2^{\text {vii }}$ & $3.2202(11)$ & $\mathrm{Si} 1-\mathrm{Gd}^{\mathrm{i}}$ & $2.9604(12)$ \\
\hline $\mathrm{Gd}-\mathrm{Si} 2$ & $3.2202(11)$ & $\mathrm{Si} 1-\mathrm{Gd}^{\mathrm{ii}}$ & $2.9604(12)$ \\
\hline $\mathrm{Gd}-\mathrm{Re} 2^{\text {viii }}$ & $3.3047(5)$ & $\mathrm{Si} 2-\mathrm{Re} 1^{\mathrm{iii}}$ & $2.4837(12)$ \\
\hline $\mathrm{Re} 1-\mathrm{Si} 1^{\mathrm{ix}}$ & $2.467(4)$ & $\mathrm{Si} 2-\operatorname{Re}^{\mathrm{xv}}$ & $2.4837(12)$ \\
\hline Re1-Si1 & $2.479(3)$ & $\mathrm{Si} 2-\mathrm{Si} 1^{\text {viii }}$ & $2.585(3)$ \\
\hline $\mathrm{Re} 1-\mathrm{Si} 2^{\mathrm{vi}}$ & $2.4838(12)$ & $\mathrm{Si} 2-\mathrm{Si} 1^{\mathrm{ii}}$ & $2.585(3)$ \\
\hline
\end{tabular}




\begin{tabular}{|c|c|c|c|}
\hline $\mathrm{Re} 1-\mathrm{Si}_{2}{ }^{\mathrm{v}}$ & $2.4838(12)$ & $\mathrm{Si} 2-\mathrm{Si} 2^{\mathrm{xvi}}$ & $2.7816(1)$ \\
\hline $\mathrm{Re} 1-\mathrm{Si} 3$ & $2.539(3)$ & $\mathrm{Si} 2-\mathrm{Si} 2^{\mathrm{vii}}$ & $2.7816(1)$ \\
\hline $\operatorname{Re} 1-\mathrm{Si}^{\mathrm{x}}$ & $2.539(3)$ & $\mathrm{Si} 2-\mathrm{Gd}^{\mathrm{iii}}$ & $3.163(2)$ \\
\hline $\operatorname{Re} 1-\operatorname{Re} 1^{x i}$ & $2.9683(6)$ & $\mathrm{Si} 2-\mathrm{Gd}^{\mathrm{xv}}$ & $3.163(2)$ \\
\hline $\operatorname{Re} 1-\operatorname{Re} 1^{\mathrm{ix}}$ & $2.9684(6)$ & $\mathrm{Si} 2-\mathrm{Gd}^{\mathrm{xii}}$ & $3.2202(11)$ \\
\hline $\operatorname{Re} 1-\mathrm{Gd}^{\mathrm{ii}}$ & $3.1442(3)$ & $\operatorname{Si} 3-\operatorname{Re} 1^{x}$ & $2.539(3)$ \\
\hline $\operatorname{Re} 1-\mathrm{Gd}^{\mathrm{i}}$ & $3.1442(3)$ & $\mathrm{Si} 3-\mathrm{Re} 1^{\mathrm{ix}}$ & $2.539(3)$ \\
\hline $\mathrm{Re} 2-\mathrm{Si}^{1 \mathrm{ii}}$ & $2.476(3)$ & $\mathrm{Si} 3-\mathrm{Re}^{\mathrm{xi}}$ & $2.539(3)$ \\
\hline $\operatorname{Re} 2-\mathrm{Si}^{\mathrm{xii}}$ & $2.476(3)$ & $\mathrm{Si} 3-\mathrm{Si}^{\mathrm{xvii}}$ & $2.707(9)$ \\
\hline $\operatorname{Re} 2-\mathrm{Si}^{\text {viii }}$ & $2.476(3)$ & $\mathrm{Si} 3-\mathrm{Gd}^{\mathrm{ii}}$ & $3.053(2)$ \\
\hline Re2-Sil & $2.476(3)$ & $\mathrm{Si} 3-\mathrm{Gd}^{\mathrm{xviii}}$ & $3.053(2)$ \\
\hline $\mathrm{Re} 2-\mathrm{Si} 2^{\mathrm{xiii}}$ & $2.662(3)$ & $\mathrm{Si} 3-\mathrm{Gd}^{\mathrm{xix}}$ & $3.053(2)$ \\
\hline $\mathrm{Re} 2-\mathrm{Si} 2$ & $2.662(3)$ & $\mathrm{Si} 3-\mathrm{Gd}^{\mathrm{xx}}$ & $3.053(2)$ \\
\hline $\mathrm{Si} 1-\mathrm{Gd}-\mathrm{Si}^{\mathrm{i}}$ & $79.48(8)$ & $\mathrm{Si} 1^{\mathrm{ii}}-\mathrm{Re} 2-\mathrm{Gd}^{\mathrm{xii}}$ & $94.36(7)$ \\
\hline $\mathrm{Si} 1-\mathrm{Gd}-\mathrm{Si} 1^{\mathrm{ii}}$ & $79.48(8)$ & $\mathrm{Si} 1^{\mathrm{xii}}-\operatorname{Re} 2-\mathrm{Gd}^{\mathrm{xii}}$ & $56.71(8)$ \\
\hline $\mathrm{Si} 1^{\mathrm{i}}-\mathrm{Gd}-\mathrm{Si} 1^{\mathrm{ii}}$ & $139.97(12)$ & $\mathrm{Si} 1^{\mathrm{vii}}-\mathrm{Re} 2-\mathrm{Gd}^{\mathrm{xii}}$ & $59.57(4)$ \\
\hline $\mathrm{Si} 1-\mathrm{Gd}-\mathrm{Si} 3^{\mathrm{iii}}$ & $152.94(8)$ & $\mathrm{Si} 1-\operatorname{Re} 2-\mathrm{Gd}^{\mathrm{xii}}$ & $168.30(6)$ \\
\hline $\mathrm{Si} 1^{\mathrm{i}}-\mathrm{Gd}-\mathrm{Si} 3^{\mathrm{iii}}$ & $127.50(10)$ & $\mathrm{Si} 2^{\mathrm{xii}}-\mathrm{Re} 2-\mathrm{Gd}^{\mathrm{xii}}$ & $115.730(10)$ \\
\hline $\mathrm{Si}^{\mathrm{ii}}-\mathrm{Gd}-\mathrm{Si} 3^{\mathrm{iii}}$ & $77.03(10)$ & $\mathrm{Si} 2-\mathrm{Re} 2-\mathrm{Gd}^{\mathrm{xii}}$ & $64.270(10)$ \\
\hline $\mathrm{Si} 1-\mathrm{Gd}-\mathrm{Si}^{\mathrm{iv}}$ & $152.94(8)$ & $\operatorname{Re} 2^{\text {xiv }}-\operatorname{Re} 2-G^{x i i}$ & $65.111(4)$ \\
\hline $\mathrm{Si} 1^{\mathrm{i}}-\mathrm{Gd}-\mathrm{Si}^{\mathrm{iv}}$ & $77.03(10)$ & $\operatorname{Re} 2^{\text {viii }-\operatorname{Re} 2-\mathrm{Gd}^{\mathrm{xii}}}$ & $114.889(4)$ \\
\hline $\mathrm{Si} 1^{\mathrm{ii}}-\mathrm{Gd}-\mathrm{Si}^{\mathrm{iv}}$ & $127.50(10)$ & $\mathrm{Gd}^{\mathrm{vii}}-\mathrm{Re} 2-\mathrm{Gd}^{\mathrm{xii}}$ & $74.405(16)$ \\
\hline $\mathrm{Si}^{\mathrm{iii}}-\mathrm{Gd}-\mathrm{Si}^{\mathrm{iv}}$ & $52.64(16)$ & $\mathrm{Si} 1^{\mathrm{ii}}-\mathrm{Re} 2-\mathrm{Gd}^{\mathrm{ii}}$ & $56.71(8)$ \\
\hline $\mathrm{Si} 1-\mathrm{Gd}-\mathrm{Re} 1^{\mathrm{ii}}$ & $105.17(3)$ & $\mathrm{Si}^{\mathrm{x}}{ }^{\mathrm{xii}}-\mathrm{Re} 2-\mathrm{Gd}^{\mathrm{ii}}$ & $94.36(7)$ \\
\hline $\mathrm{Si} 1^{\mathrm{i}-}-\mathrm{Gd}-\mathrm{Re} 1^{\mathrm{ii}}$ & $172.19(7)$ & $\mathrm{Si}^{\mathrm{viii}}{ }^{\mathrm{vi}} \mathrm{Re}-\mathrm{Gd}^{\mathrm{ii}}$ & $168.29(6)$ \\
\hline 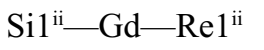 & $47.80(6)$ & $\mathrm{Si} 1-\mathrm{Re} 2-\mathrm{Gd}^{\mathrm{ii}}$ & $59.57(4)$ \\
\hline $\mathrm{Si} 3^{\mathrm{iii}}-\mathrm{Gd}-\mathrm{Re} 1^{\mathrm{ii}}$ & $48.34(7)$ & $\mathrm{Si} 2^{\mathrm{xiii}}-\mathrm{Re} 2-\mathrm{Gd}^{\mathrm{ii}}$ & $64.270(10)$ \\
\hline $\mathrm{Si}^{\mathrm{iv}}-\mathrm{Gd}-\mathrm{Re} 1^{\mathrm{ii}}$ & $96.88(8)$ & $\mathrm{Si} 2-\mathrm{Re} 2-\mathrm{Gd}^{\mathrm{ii}}$ & $115.730(10)$ \\
\hline $\mathrm{Si} 1-\mathrm{Gd}-\mathrm{Re} 1^{\mathrm{i}}$ & $105.17(3)$ & $\operatorname{Re} 2^{\mathrm{xiv}}-\mathrm{Re} 2-\mathrm{Gd}^{\mathrm{ii}}$ & $65.111(4)$ \\
\hline $\mathrm{Si} 1^{\mathrm{i}}-\mathrm{Gd}-\operatorname{Re} 1^{\mathrm{i}}$ & $47.80(6)$ & $\operatorname{Re} 2^{\text {viii }}-\operatorname{Re} 2-\mathrm{Gd}^{\mathrm{ii}}$ & $114.889(4)$ \\
\hline $\mathrm{Si} 1^{\mathrm{ii}}-\mathrm{Gd}-\mathrm{Re} 1^{\mathrm{i}}$ & $172.19(7)$ & $\mathrm{Gd}^{\mathrm{viii}}-\mathrm{Re} 2-\mathrm{Gd}^{\mathrm{ii}}$ & $128.540(19)$ \\
\hline $\mathrm{Si}^{\mathrm{iii}}-\mathrm{Gd}-\mathrm{Re} 1^{\mathrm{i}}$ & $96.88(8)$ & $\mathrm{Gd}^{\mathrm{xii}}-\mathrm{Re} 2-\mathrm{Gd}^{\mathrm{ii}}$ & $130.223(8)$ \\
\hline $\mathrm{Si}^{3}{ }^{\mathrm{iv}}-\mathrm{Gd}-\mathrm{Re}^{\mathrm{i}}$ & $48.34(7)$ & $\mathrm{Si} 1^{\mathrm{ii}-\mathrm{Re} 2-\mathrm{Gd}}$ & $59.57(4)$ \\
\hline $\operatorname{Re} 1^{i i}-\mathrm{Gd}-\operatorname{Re} 1^{\mathrm{i}}$ & $124.42(2)$ & $\mathrm{Si} 1^{\mathrm{xii}}-\mathrm{Re} 2-\mathrm{Gd}$ & $168.30(6)$ \\
\hline $\mathrm{Si} 1-\mathrm{Gd}-\mathrm{Si} 2^{\mathrm{v}}$ & $81.12(7)$ & Si $1^{\text {viii_-Re2-Gd }}$ & $94.36(7)$ \\
\hline $\mathrm{Si} 1^{\mathrm{i}}-\mathrm{Gd}-\mathrm{Si}^{\mathrm{v}}$ & $79.33(7)$ & $\mathrm{Si} 1-\mathrm{Re} 2-\mathrm{Gd}$ & $56.71(8)$ \\
\hline $\mathrm{Si}{ }^{\mathrm{ii}}-\mathrm{Gd}-\mathrm{Si}^{\mathrm{v}}$ & $129.87(7)$ & $\mathrm{Si} 2^{\text {xiii }}-\mathrm{Re} 2-\mathrm{Gd}$ & $115.731(10)$ \\
\hline $\mathrm{Si} 3^{\mathrm{iii}}-\mathrm{Gd}-\mathrm{Si}^{\mathrm{v}}$ & $104.01(5)$ & $\mathrm{Si} 2-\mathrm{Re} 2-\mathrm{Gd}$ & $64.269(10)$ \\
\hline $\mathrm{Si}^{\mathrm{iv}}-\mathrm{Gd}-\mathrm{Si}^{\mathrm{v}}$ & $81.50(4)$ & $\operatorname{Re} 2^{\text {xiv }}-\operatorname{Re} 2-G d$ & $114.888(4)$ \\
\hline $\operatorname{Re} 1^{\mathrm{ii}}-\mathrm{Gd}-\mathrm{Si}^{\mathrm{v}}$ & $95.05(3)$ & $\operatorname{Re} 2^{\mathrm{viii}-\mathrm{Re} 2-\mathrm{Gd}}$ & $65.112(4)$ \\
\hline $\mathrm{Re} 1^{\mathrm{i}}-\mathrm{Gd}-\mathrm{Si}^{\mathrm{v}}$ & $46.377(11)$ & $\mathrm{Gd}^{\text {viii }}-\mathrm{Re} 2-\mathrm{Gd}$ & $130.223(8)$ \\
\hline $\mathrm{Si} 1-\mathrm{Gd}-\mathrm{Si} 2^{\mathrm{vi}}$ & $81.12(7)$ & $\mathrm{Gd}^{\mathrm{xii}}-\mathrm{Re} 2-\mathrm{Gd}$ & $128.540(19)$ \\
\hline $\mathrm{Si} 1^{\mathrm{i}}-\mathrm{Gd}-\mathrm{Si} 2^{\mathrm{vi}}$ & $129.87(7)$ & $\mathrm{Gd}^{\mathrm{ii}}-\mathrm{Re} 2-\mathrm{Gd}$ & $74.406(16)$ \\
\hline $\mathrm{Si} 1^{\mathrm{ii}}-\mathrm{Gd}-\mathrm{Si} 2^{\mathrm{vi}}$ & $79.33(7)$ & $\operatorname{Re} 1^{\mathrm{xi}}-\mathrm{Si} 1-\operatorname{Re} 2$ & $122.21(11)$ \\
\hline $\mathrm{Si} 3^{\mathrm{iii}}-\mathrm{Gd}-\mathrm{Si} 2^{\mathrm{vi}}$ & $81.50(4)$ & $\operatorname{Re} 1^{x i}-\operatorname{Si} 1-\operatorname{Re} 2^{\text {viii }}$ & $122.21(11)$ \\
\hline $\mathrm{Si}^{\mathrm{iv}}-\mathrm{Gd}-\mathrm{Si}^{\mathrm{vi}}$ & $104.01(5)$ & $\operatorname{Re} 2-\operatorname{Si} 1-\operatorname{Re} 2^{\text {viii }}$ & $68.34(9)$ \\
\hline $\operatorname{Re} 1^{1 i}-\mathrm{Gd}-\mathrm{Si}^{\mathrm{iv}}$ & $46.377(11)$ & $\operatorname{Re} 1^{\mathrm{xi}}-\mathrm{Si} 1-\operatorname{Re} 1$ & $73.76(10)$ \\
\hline
\end{tabular}




\begin{tabular}{|c|c|c|c|}
\hline $\mathrm{Re} 1^{\mathrm{i}}-\mathrm{Gd}-\mathrm{Si} 2^{\mathrm{vi}}$ & $95.05(3)$ & $\operatorname{Re} 2-\operatorname{Si} 1-\operatorname{Re} 1$ & $139.17(9)$ \\
\hline $\mathrm{Si} 2^{\mathrm{v}}-\mathrm{Gd}-\mathrm{Si} 2^{\mathrm{vi}}$ & $52.16(4)$ & $\operatorname{Re} 2^{\text {viii }}-\operatorname{Si} 1-\operatorname{Re} 1$ & $139.17(9)$ \\
\hline $\mathrm{Si} 1-\mathrm{Gd}-\mathrm{Si} 2^{\mathrm{vii}}$ & $94.12(8)$ & $\mathrm{Re} 1^{\mathrm{xi}}-\mathrm{Si} 1-\mathrm{Si} 2^{\text {viii }}$ & $58.84(9)$ \\
\hline $\mathrm{Si} 1^{\mathrm{i}}-\mathrm{Gd}-\mathrm{Si}^{\mathrm{vii}}$ & $49.23(7)$ & $\mathrm{Re} 2-\mathrm{Si} 1-\mathrm{Si} 2^{\text {viii }}$ & $99.03(11)$ \\
\hline $\mathrm{Si} 1^{\mathrm{ii}}-\mathrm{Gd}-\mathrm{Si} 2^{\mathrm{vii}}$ & $99.13(6)$ & $\mathrm{Re} 2^{\text {viii }}-\mathrm{Si} 1-\mathrm{Si} 2^{\text {viii }}$ & $63.42(8)$ \\
\hline $\mathrm{Si}^{\mathrm{iii}}-\mathrm{Gd}-\mathrm{Si} 2^{\mathrm{vii}}$ & $102.67(6)$ & $\mathrm{Re} 1-\mathrm{Si} 1-\mathrm{Si} 2^{\text {viii }}$ & $119.61(12)$ \\
\hline $\mathrm{Si}^{\mathrm{iv}}-\mathrm{Gd}-\mathrm{Si} 2^{\mathrm{vii}}$ & $80.58(5)$ & $\operatorname{Re} 1^{x i}-\operatorname{Si} 1-S i 2^{x i i i}$ & $58.84(9)$ \\
\hline $\mathrm{Re} 1^{\mathrm{ii}}-\mathrm{Gd}-\mathrm{Si} 2^{\mathrm{vii}}$ & $135.25(3)$ & $\mathrm{Re} 2-\mathrm{Si} 1-\mathrm{Si} 2^{\mathrm{xiii}}$ & $63.42(8)$ \\
\hline $\mathrm{Re} 1^{\mathrm{i}}-\mathrm{Gd}-\mathrm{Si} 2^{\mathrm{vii}}$ & $86.902(11)$ & $\mathrm{Re} 2^{\mathrm{viii}}-\mathrm{Si} 1-\mathrm{Si} 2^{\mathrm{xiii}}$ & $99.03(11)$ \\
\hline $\mathrm{Si} 2^{\mathrm{v}}-\mathrm{Gd}-\mathrm{Si} 2^{\mathrm{vii}}$ & $128.074(8)$ & $\mathrm{Re} 1-\mathrm{Si} 1-\mathrm{Si} 2^{\mathrm{xiii}}$ & $119.61(12)$ \\
\hline $\mathrm{Si} 2^{\mathrm{vi}}-\mathrm{Gd}-\mathrm{Si}^{\mathrm{vii}}$ & $175.18(3)$ & 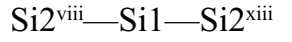 & $65.09(9)$ \\
\hline $\mathrm{Si} 1-\mathrm{Gd}-\mathrm{Si} 2$ & $94.12(8)$ & $\operatorname{Re} 1^{x i}-\operatorname{Si} 1-G d$ & $156.27(14)$ \\
\hline $\mathrm{Si} 11^{\mathrm{i}}-\mathrm{Gd}-\mathrm{Si} 2$ & $99.13(6)$ & $\mathrm{Re} 2-\mathrm{Si} 1-\mathrm{Gd}$ & $76.51(9)$ \\
\hline $\mathrm{Si} 1{ }^{\mathrm{ii}}-\mathrm{Gd}-\mathrm{Si} 2$ & $49.23(7)$ & $\mathrm{Re}^{\mathrm{viii}}-\mathrm{Si} 1-\mathrm{Gd}$ & $76.51(9)$ \\
\hline $\mathrm{Si} 3^{\mathrm{iii}}-\mathrm{Gd}-\mathrm{Si} 2$ & $80.58(5)$ & $\mathrm{Re} 1-\mathrm{Si} 1-\mathrm{Gd}$ & $82.50(10)$ \\
\hline $\mathrm{Si} 3^{\text {iv }}-\mathrm{Gd}-\mathrm{Si} 2$ & $102.67(6)$ & 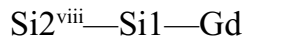 & $137.86(11)$ \\
\hline $\operatorname{Re} 1^{\mathrm{ii}}-\mathrm{Gd}-\mathrm{Si} 2$ & $86.902(11)$ & $\mathrm{Si} 2^{\text {xiii }-S i 1}-\mathrm{Gd}$ & $137.86(11)$ \\
\hline $\mathrm{Re} 1{ }^{\mathrm{i}-\mathrm{Gd}}-\mathrm{Si} 2$ & $135.25(3)$ & $\mathrm{Re}^{\mathrm{xi}}-\mathrm{Si} 1-\mathrm{Gd}^{\mathrm{i}}$ & $84.88(7)$ \\
\hline $\mathrm{Si} 2^{\mathrm{v}}-\mathrm{Gd}-\mathrm{Si} 2$ & $175.18(3)$ & $\mathrm{Re} 2-\mathrm{Si} 1-\mathrm{Gd}^{\mathrm{i}}$ & $141.66(11)$ \\
\hline $\mathrm{Si} 2^{\mathrm{vi}}-\mathrm{Gd}-\mathrm{Si} 2$ & $128.075(8)$ & $\operatorname{Re} 2^{\text {viii }-S i 1}-G^{i}$ & $74.27(2)$ \\
\hline $\mathrm{Si} 2^{\text {vii }}-\mathrm{Gd}-\mathrm{Si} 2$ & $51.18(2)$ & $\mathrm{Re} 1-\mathrm{Si} 1-\mathrm{Gd}^{\mathrm{i}}$ & $69.99(6)$ \\
\hline $\mathrm{Si} 1-\mathrm{Gd}-\mathrm{Re} 2^{\text {viii }}$ & $46.77(6)$ & $\mathrm{Si} 2^{\text {viii }}-\mathrm{Si} 1-\mathrm{Gd}^{\mathrm{i}}$ & $70.63(3)$ \\
\hline $\mathrm{Si} 1^{\mathrm{i}}-\mathrm{Gd}-\mathrm{Re} 2^{\text {viii }}$ & $46.16(6)$ & $\mathrm{Si} 2^{\mathrm{xiii}}-\mathrm{Si} 1-\mathrm{Gd}^{\mathrm{i}}$ & $132.78(12)$ \\
\hline $\mathrm{Si} 1^{\mathrm{ii}}-\mathrm{Gd}-\mathrm{Re} 2^{\mathrm{viii}}$ & $95.63(6)$ & $\mathrm{Gd}-\mathrm{Si} 1-\mathrm{Gd}^{\mathrm{i}}$ & $87.06(7)$ \\
\hline $\mathrm{Si} 3^{\mathrm{iii}}-\mathrm{Gd}-\mathrm{Re} 2^{\mathrm{viii}}$ & $149.01(2)$ & $\operatorname{Re} 1^{\mathrm{xi}}-\mathrm{Si} 1-\mathrm{Gd}^{\mathrm{ii}}$ & $84.88(7)$ \\
\hline $\mathrm{Si}^{\mathrm{iv}}-\mathrm{Gd}-\mathrm{Re} 2^{\mathrm{viii}}$ & $118.95(7)$ & $\operatorname{Re} 2-\mathrm{Si} 1-\mathrm{Gd}^{\mathrm{ii}}$ & $74.27(2)$ \\
\hline $\operatorname{Re} 1^{\mathrm{ii}}-\mathrm{Gd}-\mathrm{Re} 2^{\text {viii }}$ & $141.358(16)$ & $\operatorname{Re} 2^{\mathrm{viii}}-\mathrm{Si} 1-\mathrm{Gd}^{\mathrm{ii}}$ & $141.66(11)$ \\
\hline $\operatorname{Re} 1^{\mathrm{i}}-\mathrm{Gd}-\operatorname{Re} 2^{\text {viii }}$ & $92.088(9)$ & $\operatorname{Re} 1-\mathrm{Si} 1-\mathrm{Gd}^{\mathrm{ii}}$ & $69.99(6)$ \\
\hline $\mathrm{Si} 2^{\mathrm{v}}-\mathrm{Gd}-\mathrm{Re} 2^{\mathrm{viii}}$ & $103.62(3)$ & $\mathrm{Si} 2^{\mathrm{viii}}-\mathrm{Si} 1-\mathrm{Gd}^{\mathrm{ii}}$ & $132.78(12)$ \\
\hline $\mathrm{Si} 2^{\mathrm{vi}}-\mathrm{Gd}-\mathrm{Re} 2^{\mathrm{viii}}$ & $127.27(3)$ & $\mathrm{Si} 2^{\mathrm{xiii}}-\mathrm{Si} 1-\mathrm{Gd}^{\mathrm{ii}}$ & $70.63(3)$ \\
\hline $\mathrm{Si} 2^{\mathrm{vii}}-\mathrm{Gd}-\mathrm{Re} 2^{\mathrm{viii}}$ & $48.14(5)$ & $\mathrm{Gd}-\mathrm{Si} 1-\mathrm{Gd}^{\mathrm{ii}}$ & $87.06(7)$ \\
\hline $\mathrm{Si} 2-\mathrm{Gd}-\mathrm{Re} 2^{\mathrm{viii}}$ & $72.31(3)$ & $\mathrm{Gd}^{\mathrm{i}}-\mathrm{Si} 1-\mathrm{Gd}^{\mathrm{ii}}$ & $139.97(12)$ \\
\hline $\mathrm{Si} 1^{\mathrm{ix}}-\mathrm{Re} 1-\mathrm{Si} 1$ & $163.76(10)$ & $\operatorname{Re} 1^{\mathrm{iii}}-\mathrm{Si} 2-\operatorname{Re} 1^{\mathrm{xv}}$ & $131.08(12)$ \\
\hline $\mathrm{Si} 1^{\mathrm{ix}}-\mathrm{Re} 1-\mathrm{Si}^{\mathrm{vi}}$ & $62.95(8)$ & $\operatorname{Re} 1^{\mathrm{iii}}-\mathrm{Si} 2-\mathrm{Si} 1^{\text {viii }}$ & $170.53(14)$ \\
\hline $\mathrm{Si} 1-\mathrm{Re} 1-\mathrm{Si}^{\mathrm{vi}}$ & $104.06(9)$ & $\mathrm{Re} 1^{\mathrm{xv}}-\mathrm{Si} 2-\mathrm{Si} 1^{\mathrm{viii}}$ & $58.21(7)$ \\
\hline $\mathrm{Si} 1^{\mathrm{ix}}-\mathrm{Re} 1-\mathrm{Si}^{2}{ }^{\mathrm{v}}$ & $62.95(8)$ & $\mathrm{Re} 1^{\mathrm{iii}}-\mathrm{Si} 2-\mathrm{Si} 1^{\mathrm{ii}}$ & $58.21(7)$ \\
\hline $\mathrm{Si} 1-\mathrm{Re} 1-\mathrm{Si}^{\mathrm{v}}$ & $104.06(9)$ & $\mathrm{Re} 1^{\mathrm{xv}}-\mathrm{Si} 2-\mathrm{Si} 1^{1 \mathrm{ii}}$ & $170.53(14)$ \\
\hline $\mathrm{Si} 2^{\mathrm{vi}}-\mathrm{Re} 1-\mathrm{Si}^{\mathrm{v}}$ & $68.11(4)$ & 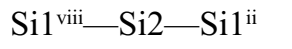 & $112.58(18)$ \\
\hline $\mathrm{Si} 1{ }^{\mathrm{ix}}-\mathrm{Re} 1-\mathrm{Si} 3$ & $96.85(6)$ & $\operatorname{Re} 1^{\mathrm{iii}}-\mathrm{Si} 2-\operatorname{Re} 2$ & $114.46(6)$ \\
\hline $\mathrm{Si} 1-\mathrm{Re} 1-\mathrm{Si} 3$ & $96.56(7)$ & $\mathrm{Re}^{\mathrm{xv}}-\mathrm{Si} 2-\operatorname{Re} 2$ & $114.46(6)$ \\
\hline $\mathrm{Si} 2^{\mathrm{vi}}-\mathrm{Re} 1-\mathrm{Si} 3$ & $107.81(8)$ & 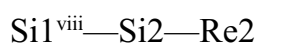 & $56.29(9)$ \\
\hline $\mathrm{Si} 2^{v}-\mathrm{Re} 1-\mathrm{Si} 3$ & $159.37(6)$ & $\mathrm{Si} 1{ }^{\mathrm{ii}}-\mathrm{Si} 2-\mathrm{Re} 2$ & $56.29(9)$ \\
\hline $\mathrm{Si} 1^{\mathrm{ix}}-\mathrm{Re} 1-\mathrm{Si}{ }^{\mathrm{x}}$ & $96.85(6)$ & $\mathrm{Re} 1^{\mathrm{iii}}-\mathrm{Si} 2-\mathrm{Si} 2^{\mathrm{xvi}}$ & $55.947(19)$ \\
\hline $\operatorname{Si} 1-\operatorname{Re} 1-\mathrm{Si}^{\mathrm{x}}$ & $96.56(7)$ & $\mathrm{Re} 1^{\mathrm{xv}}-\mathrm{Si} 2-\mathrm{Si} 2^{\mathrm{xvi}}$ & $124.054(19)$ \\
\hline $\mathrm{Si} 2^{\mathrm{vi}}-\mathrm{Re} 1-\mathrm{Si}{ }^{\mathrm{x}}$ & $159.37(6)$ & $\mathrm{Si} 1^{\mathrm{viii}}-\mathrm{Si} 2-\mathrm{Si} 2^{\mathrm{xvi}}$ & $122.55(5)$ \\
\hline $\mathrm{Si} 2^{\mathrm{v}}-\mathrm{Re} 1-\mathrm{Si}^{\mathrm{x}}$ & $107.81(8)$ & $\mathrm{Si} 1^{\mathrm{ii}}-\mathrm{Si} 2-\mathrm{Si} 2^{\mathrm{xvi}}$ & $57.45(5)$ \\
\hline $\mathrm{Si} 3-\mathrm{Re} 1-\mathrm{Si}^{\mathrm{x}}$ & $68.46(18)$ & $\mathrm{Re} 2-\mathrm{Si} 2-\mathrm{Si} 2^{\mathrm{xvi}}$ & 90.0 \\
\hline $\mathrm{Si} 1^{\mathrm{ix}}-\operatorname{Re} 1-\operatorname{Re} 1^{\mathrm{xi}}$ & $143.30(8)$ & $\mathrm{Re} 1^{\mathrm{iii}}-\mathrm{Si} 2-\mathrm{Si} 2^{\mathrm{vii}}$ & $124.054(19)$ \\
\hline
\end{tabular}




\begin{tabular}{|c|c|}
\hline $\operatorname{Si} 1-\operatorname{Re} 1-\operatorname{Re} 1^{\mathrm{xi}}$ & $52.94(8)$ \\
\hline 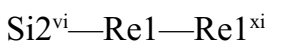 & $141.12(5)$ \\
\hline $\mathrm{Si} 2^{\mathrm{v}}-\operatorname{Re} 1-\operatorname{Re} 1^{\mathrm{xi}}$ & $141.12(5)$ \\
\hline Si3-Re1-Re1 ${ }^{x i}$ & $54.22(4)$ \\
\hline $\operatorname{Si} 3^{x}-\operatorname{Re} 1-\operatorname{Re} 1^{x i}$ & $54.22(4)$ \\
\hline $\operatorname{Si} 1^{\mathrm{ix}}-\operatorname{Re} 1-\operatorname{Re} 1^{\mathrm{ix}}$ & $53.30(8)$ \\
\hline $\operatorname{Si} 1-\operatorname{Re} 1-\operatorname{Re} 1^{\mathrm{ix}}$ & $142.94(8)$ \\
\hline 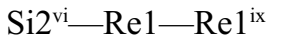 & $106.47(5)$ \\
\hline $\mathrm{Si} 2^{\mathrm{v}}-\operatorname{Re} 1-\operatorname{Re} 1^{\mathrm{ix}}$ & $106.47(5)$ \\
\hline $\mathrm{Si} 3-\operatorname{Re} 1-\mathrm{Re}^{\mathrm{ix}}$ & $54.22(4)$ \\
\hline $\operatorname{Si} 3^{x}-\operatorname{Re} 1-\operatorname{Re} 1^{i x}$ & $54.22(4)$ \\
\hline $\operatorname{Re} 1^{x i}-\operatorname{Re} 1-\operatorname{Re} 1^{\mathrm{ix}}$ & 90.0 \\
\hline $\mathrm{Si} 1^{\mathrm{ix}}-\mathrm{Re} 1-\mathrm{Gd}^{\mathrm{ii}}$ & $116.473(18)$ \\
\hline $\mathrm{Si} 1-\operatorname{Re} 1-\mathrm{Gd}^{\mathrm{ii}}$ & $62.214(12)$ \\
\hline $\mathrm{Si} 2^{\mathrm{vi}}-\mathrm{Re} 1-\mathrm{Gd}^{\mathrm{ii}}$ & $67.22(4)$ \\
\hline $\mathrm{Si} 2^{\mathrm{v}}-\mathrm{Re} 1-\mathrm{Gd}^{\mathrm{ii}}$ & $127.12(2)$ \\
\hline $\mathrm{Si} 3-\mathrm{Re} 1-\mathrm{Gd}^{\mathrm{ii}}$ & $63.95(8)$ \\
\hline $\mathrm{Si} 3^{\mathrm{x}}-\mathrm{Re} 1-\mathrm{Gd}^{\mathrm{ii}}$ & $123.79(8)$ \\
\hline $\operatorname{Re} 1^{\mathrm{xi}}-\operatorname{Re} 1-\mathrm{Gd}^{\mathrm{ii}}$ & $73.988(16)$ \\
\hline $\operatorname{Re} 1^{\mathrm{ix}}-\operatorname{Re} 1-\mathrm{Gd}^{\mathrm{ii}}$ & $112.077(11)$ \\
\hline $\mathrm{Si} 1^{\mathrm{ix}}-\mathrm{Re} 1-\mathrm{Gd}^{\mathrm{i}}$ & $116.473(18)$ \\
\hline $\mathrm{Si} 1-\mathrm{Re} 1-\mathrm{Gd}^{\mathrm{i}}$ & $62.214(12)$ \\
\hline 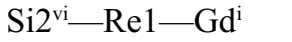 & $127.12(2)$ \\
\hline $\mathrm{Si} 2^{\mathrm{v}}-\mathrm{Re} 1-\mathrm{Gd}^{\mathrm{i}}$ & $67.22(4)$ \\
\hline $\mathrm{Si} 3-\mathrm{Re} 1-\mathrm{Gd}^{\mathrm{i}}$ & $123.79(8)$ \\
\hline $\mathrm{Si}^{\mathrm{x}}-\mathrm{Re} 1-\mathrm{Gd}^{\mathrm{i}}$ & $63.95(8)$ \\
\hline $\operatorname{Re}^{1 \mathrm{x}}-\operatorname{Re} 1-\mathrm{Gd}^{\mathrm{i}}$ & $73.988(16)$ \\
\hline $\operatorname{Re} 1^{1 \mathrm{x}}-\operatorname{Re} 1-\mathrm{Gd}^{\mathrm{i}}$ & $112.077(11)$ \\
\hline $\mathrm{Gd}^{\mathrm{ii}}-\mathrm{Re} 1-\mathrm{Gd}^{\mathrm{i}}$ & $124.42(2)$ \\
\hline $\mathrm{Si} 1^{\mathrm{ix}}-\mathrm{Re} 1-\mathrm{Gd}$ & $110.57(8)$ \\
\hline $\mathrm{Si} 1-\mathrm{Re} 1-\mathrm{Gd}$ & $53.19(8)$ \\
\hline $\mathrm{Si} 2^{\mathrm{vi}}-\mathrm{Re} 1-\mathrm{Gd}$ & $60.75(5)$ \\
\hline $\mathrm{Si} 2^{\mathrm{v}}-\mathrm{Re} 1-\mathrm{Gd}$ & $60.75(5)$ \\
\hline $\mathrm{Si} 3-\mathrm{Re} 1-\mathrm{Gd}$ & $136.39(6)$ \\
\hline $\mathrm{Si} 3^{x}-\operatorname{Re} 1-\mathrm{Gd}$ & $136.39(6)$ \\
\hline $\operatorname{Re} 1^{x i}-\operatorname{Re} 1-G d$ & $106.13(2)$ \\
\hline $\operatorname{Re} 1^{1 x}-\operatorname{Re} 1-G d$ & $163.87(2)$ \\
\hline $\mathrm{Gd}^{\mathrm{ii}}-\mathrm{Re} 1-\mathrm{Gd}$ & $73.474(15)$ \\
\hline $\mathrm{Gd}^{\mathrm{i}}-\mathrm{Re} 1-\mathrm{Gd}$ & $73.474(15)$ \\
\hline $\mathrm{Si}^{\mathrm{ii}}-\mathrm{Re} 2-\mathrm{Si}^{\mathrm{xii}}$ & $111.66(9)$ \\
\hline $\mathrm{Si} 1^{\mathrm{ii}}-\mathrm{Re} 2-\mathrm{Si}^{\mathrm{viii}}$ & $120.57(15)$ \\
\hline $\mathrm{Si} 1^{\mathrm{xii}}-\mathrm{Re} 2-\mathrm{Si} 1^{\text {viii }}$ & $97.03(13)$ \\
\hline 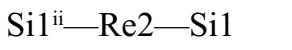 & $97.03(13)$ \\
\hline $\mathrm{Si}^{\mathrm{xii}}-\mathrm{Re} 2-\mathrm{Si} 1$ & $120.57(15)$ \\
\hline Si1 ${ }^{\text {viii-Re2-Si1 }}$ & $111.66(9)$ \\
\hline $\mathrm{Si} 1^{\mathrm{ii}}-\mathrm{Re} 2-\mathrm{Si} 2^{\mathrm{xii}}$ & $119.72(8)$ \\
\hline $\mathrm{Si} 1^{\mathrm{xii}}-\mathrm{Re} 2-\mathrm{Si} 2^{\mathrm{xiii}}$ & $60.28(8)$ \\
\hline $\mathrm{Si} 1^{\text {viii- }}$ Re2-Si2 $2^{\text {xii }}$ & $119.72(8)$ \\
\hline
\end{tabular}

\begin{tabular}{|c|c|}
\hline $\operatorname{Re} 1^{\mathrm{xv}}-\mathrm{Si} 2-\mathrm{Si} 2^{\mathrm{vii}}$ & $55.947(19)$ \\
\hline $\mathrm{Si} 1^{\mathrm{viii}}-\mathrm{Si} 2-\mathrm{Si}^{\mathrm{vii}}$ & $57.45(5)$ \\
\hline $\mathrm{Si} 1^{\mathrm{ii}}-\mathrm{Si} 2-\mathrm{Si} 2^{\mathrm{vii}}$ & $122.55(5)$ \\
\hline $\mathrm{Re} 2-\mathrm{Si} 2-\mathrm{Si} 2^{\mathrm{vii}}$ & 90.0 \\
\hline $\mathrm{Si} 2^{\mathrm{xvi}}-\mathrm{Si} 2-\mathrm{Si}^{2 \mathrm{vi}}$ & 180.0 \\
\hline $\operatorname{Re} 1^{\mathrm{iii}}-\mathrm{Si} 2-\mathrm{Gd}^{\mathrm{iii}}$ & $76.01(6)$ \\
\hline $\mathrm{Re}^{\mathrm{xv}}-\mathrm{Si} 2-\mathrm{Gd}^{\mathrm{iii}}$ & $66.41(5)$ \\
\hline $\mathrm{Si}^{\mathrm{viii}}-\mathrm{Si} 2-\mathrm{Gd}^{\mathrm{iii}}$ & $112.27(8)$ \\
\hline $\mathrm{Si} 1^{\mathrm{ii}}-\mathrm{Si} 2-\mathrm{Gd}^{\mathrm{iii}}$ & $118.78(6)$ \\
\hline $\mathrm{Re} 2-\mathrm{Si} 2-\mathrm{Gd}^{\mathrm{iii}}$ & $140.83(3)$ \\
\hline $\mathrm{Si} 2^{\mathrm{xvi}}-\mathrm{Si} 2-\mathrm{Gd}^{\mathrm{iii}}$ & $63.919(19)$ \\
\hline $\mathrm{Si} 2^{\mathrm{vii}}-\mathrm{Si} 2-\mathrm{Gd}^{\mathrm{iii}}$ & $116.083(19)$ \\
\hline $\mathrm{Re} 1^{\mathrm{iii}}-\mathrm{Si} 2-\mathrm{Gd}^{\mathrm{xv}}$ & $66.41(5)$ \\
\hline $\mathrm{Re}^{\mathrm{xv}}-\mathrm{Si} 2-\mathrm{Gd}^{\mathrm{xv}}$ & $76.01(6)$ \\
\hline $\mathrm{Si} 1^{\mathrm{viii}}-\mathrm{Si} 2-\mathrm{Gd}^{\mathrm{xv}}$ & $118.78(6)$ \\
\hline $\mathrm{Si} 1^{\mathrm{ii}}-\mathrm{Si} 2-\mathrm{Gd}^{\mathrm{xv}}$ & $112.27(8)$ \\
\hline $\operatorname{Re} 2-\mathrm{Si} 2-\mathrm{Gd}^{\mathrm{xv}}$ & $140.83(3)$ \\
\hline $\mathrm{Si} 2^{\mathrm{xvi}}-\mathrm{Si} 2-\mathrm{Gd}^{\mathrm{xv}}$ & $116.083(19)$ \\
\hline $\mathrm{Si} 2^{\mathrm{vii}}-\mathrm{Si} 2-\mathrm{Gd}^{\mathrm{xv}}$ & $63.919(19)$ \\
\hline $\mathrm{Gd}^{\mathrm{iii}}-\mathrm{Si} 2-\mathrm{Gd}^{\mathrm{xv}}$ & $78.35(7)$ \\
\hline $\operatorname{Re} 1^{i i i}-\mathrm{Si} 2-\mathrm{Gd}$ & $79.226(13)$ \\
\hline $\operatorname{Re} 1^{\mathrm{xv}}-\mathrm{Si} 2-\mathrm{Gd}$ & $120.171(7)$ \\
\hline 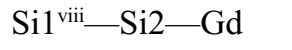 & $94.29(9)$ \\
\hline $\mathrm{Si} 1{ }^{\mathrm{ii}}-\mathrm{Si} 2-\mathrm{Gd}$ & $60.14(5)$ \\
\hline $\mathrm{Re} 2-\mathrm{Si} 2-\mathrm{Gd}$ & $67.59(5)$ \\
\hline $\mathrm{Si} 2^{\mathrm{xvi}}-\mathrm{Si} 2-\mathrm{Gd}$ & $115.588(10)$ \\
\hline $\mathrm{Si} 2^{\mathrm{vii}}-\mathrm{Si} 2-\mathrm{Gd}$ & $64.411(10)$ \\
\hline $\mathrm{Gd}^{\mathrm{iii}}-\mathrm{Si} 2-\mathrm{Gd}$ & $148.88(7)$ \\
\hline $\mathrm{Gd}^{\mathrm{xv}}-\mathrm{Si} 2-\mathrm{Gd}$ & $74.63(2)$ \\
\hline $\mathrm{Re} 1^{\mathrm{iii}}-\mathrm{Si} 2-\mathrm{Gd}^{\mathrm{xii}}$ & $120.171(7)$ \\
\hline $\mathrm{Re}^{\mathrm{xv}}-\mathrm{Si} 2-\mathrm{Gd}^{\mathrm{xii}}$ & $79.226(13)$ \\
\hline $\mathrm{Si} 1^{\mathrm{viii}}-\mathrm{Si} 2-\mathrm{Gd}^{\mathrm{xii}}$ & $60.14(5)$ \\
\hline $\mathrm{Si} 1^{\mathrm{ii}}-\mathrm{Si} 2-\mathrm{Gd}^{\mathrm{xii}}$ & $94.29(9)$ \\
\hline $\mathrm{Re} 2-\mathrm{Si} 2-\mathrm{Gd}^{\mathrm{xii}}$ & $67.59(5)$ \\
\hline $\mathrm{Si} 2^{\mathrm{xvi}}-\mathrm{Si} 2-\mathrm{Gd}^{\mathrm{xii}}$ & $64.411(10)$ \\
\hline $\mathrm{Si} 2^{\mathrm{vii}}-\mathrm{Si} 2-\mathrm{Gd}^{\mathrm{xii}}$ & $115.588(10)$ \\
\hline $\mathrm{Gd}^{\mathrm{dii}}-\mathrm{Si} 2-\mathrm{Gd}^{\mathrm{xii}}$ & $74.63(2)$ \\
\hline $\mathrm{Gd}^{\mathrm{xv}}-\mathrm{Si} 2-\mathrm{Gd}^{\mathrm{dii}}$ & $148.88(7)$ \\
\hline $\mathrm{Gd}-\mathrm{Si} 2-\mathrm{Gd}^{\mathrm{xii}}$ & $135.19(9)$ \\
\hline $\operatorname{Re} 1-\mathrm{Si} 3-\operatorname{Re} 1^{x}$ & $111.54(18)$ \\
\hline $\operatorname{Re} 1-\mathrm{Si3}-\operatorname{Re} 1^{1 \mathrm{x}}$ & $71.55(9)$ \\
\hline $\operatorname{Re} 1^{x}-S i 3-\operatorname{Re} 1^{i x}$ & $71.55(9)$ \\
\hline $\operatorname{Re} 1-\mathrm{Si} 3-\operatorname{Re} 1^{\mathrm{xi}}$ & $71.55(9)$ \\
\hline $\operatorname{Re} 1^{x}-S i 3-\operatorname{Re} 1^{x i}$ & $71.55(9)$ \\
\hline $\operatorname{Re} 1^{i x}-S i 3-\operatorname{Re} 1^{x i}$ & $111.54(18)$ \\
\hline $\mathrm{Re} 1-\mathrm{Si} 3-\mathrm{Si} 3^{\text {xvii }}$ & $124.23(9)$ \\
\hline $\mathrm{Re}^{\mathrm{x}}-\mathrm{Si} 3-\mathrm{Si}^{\mathrm{xvii}}$ & $124.23(9)$ \\
\hline $\mathrm{Re} 1^{\mathrm{ix}}-\mathrm{Si} 3-\mathrm{Si} 3^{\mathrm{xvii}}$ & $124.23(9)$ \\
\hline
\end{tabular}




\begin{tabular}{|c|c|c|c|}
\hline $\mathrm{Si} 1-\mathrm{Re} 2-\mathrm{Si} 2^{\mathrm{xiii}}$ & $60.28(8)$ & $\mathrm{Re} 1^{\mathrm{xi}}-\mathrm{Si} 3-\mathrm{Si}^{\mathrm{xvii}}$ & $124.23(9)$ \\
\hline $\mathrm{Si}_{1}{ }^{\mathrm{ii}}-\mathrm{Re} 2-\mathrm{Si} 2$ & $60.28(8)$ & $\mathrm{Re} 1-\mathrm{Si} 3-\mathrm{Gd}^{\mathrm{ii}}$ & $67.712(11)$ \\
\hline $\mathrm{Si} 1{ }^{\mathrm{xii}-\mathrm{Re} 2-\mathrm{Si} 2}$ & $119.72(8)$ & $\mathrm{Re} 1^{x}-\mathrm{Si} 3-\mathrm{Gd}^{\mathrm{ii}}$ & $151.41(5)$ \\
\hline $\mathrm{Si}^{\mathrm{viii}}-\mathrm{Re} 2-\mathrm{Si} 2$ & $60.28(8)$ & $\operatorname{Re} 1^{\mathrm{ix}}-\mathrm{Si} 3-\mathrm{Gd}^{\mathrm{ii}}$ & $129.93(3)$ \\
\hline $\mathrm{Si} 1-\mathrm{Re} 2-\mathrm{Si} 2$ & $119.72(8)$ & $\operatorname{Re} 1^{\mathrm{xi}}-\mathrm{Si} 3-\mathrm{Gd}^{\mathrm{ii}}$ & $81.779(12)$ \\
\hline $\mathrm{Si} 2^{\text {xiii }-\mathrm{Re} 2-\mathrm{Si} 2}$ & 180.0 & $\mathrm{Si} 3^{\mathrm{xvii}}-\mathrm{Si} 3-\mathrm{Gd}^{\mathrm{ii}}$ & $63.68(8)$ \\
\hline $\mathrm{Si} 1^{\mathrm{ii}}-\operatorname{Re} 2-\operatorname{Re} 2^{\mathrm{xiv}}$ & $55.83(4)$ & $\mathrm{Re} 1-\mathrm{Si} 3-\mathrm{Gd}^{\mathrm{xviii}}$ & $81.779(12)$ \\
\hline $\operatorname{Si} 1^{x i i}-\operatorname{Re} 2-\operatorname{Re} 2^{x i v}$ & $55.83(4)$ & $\mathrm{Re}^{\mathrm{x}}-\mathrm{Si} 3-\mathrm{Gd}^{\mathrm{xviii}}$ & $129.93(3)$ \\
\hline $\mathrm{Si}^{\mathrm{viii}}-\operatorname{Re} 2-\operatorname{Re} 2^{\mathrm{xiv}}$ & $124.17(4)$ & $\operatorname{Re} 1^{\mathrm{ix}}-\mathrm{Si} 3-\mathrm{Gd}^{\mathrm{xviii}}$ & $67.712(11)$ \\
\hline $\mathrm{Si} 1-\operatorname{Re} 2-\operatorname{Re} 2^{\text {xiv }}$ & $124.17(4)$ & $\mathrm{Re} 1^{\mathrm{xi}}-\mathrm{Si} 3-\mathrm{Gd}^{\mathrm{xviii}}$ & $151.41(5)$ \\
\hline $\mathrm{Si} 2^{\mathrm{xiii}}-\operatorname{Re} 2-\operatorname{Re} 2^{\mathrm{xiv}}$ & 90.0 & $\mathrm{Si} 3^{\mathrm{xvii}}-\mathrm{Si} 3-\mathrm{Gd}^{\mathrm{xviii}}$ & $63.68(8)$ \\
\hline $\mathrm{Si} 2-\mathrm{Re} 2-\mathrm{Re} 2^{\mathrm{xiv}}$ & 90.0 & $\mathrm{Gd}^{\mathrm{ii}}-\mathrm{Si} 3-\mathrm{Gd}^{\mathrm{xviii}}$ & $78.66(6)$ \\
\hline $\mathrm{Si} 1^{\mathrm{ii}}-\mathrm{Re} 2-\mathrm{Re} 2^{\mathrm{viii}}$ & $124.17(4)$ & $\operatorname{Re} 1-\mathrm{Si} 3-\mathrm{Gd}^{\mathrm{xix}}$ & $151.41(5)$ \\
\hline $\mathrm{Si}^{\mathrm{xii}}-\mathrm{Re} 2-\operatorname{Re} 2^{\mathrm{viii}}$ & $124.17(4)$ & $\operatorname{Re} 1^{x}-\mathrm{Si} 3-\mathrm{Gd}^{\mathrm{xix}}$ & $67.712(11)$ \\
\hline $\mathrm{Si}^{\mathrm{viii}}-\mathrm{Re} 2-\mathrm{Re} 2^{\mathrm{viii}}$ & $55.83(4)$ & $\operatorname{Re} 1^{\mathrm{ix}}-\mathrm{Si} 3-\mathrm{Gd}^{\mathrm{xix}}$ & $81.779(12)$ \\
\hline Si1-Re2-Re2 $2^{\text {viii }}$ & $55.83(4)$ & $\operatorname{Re} 1^{\mathrm{xi}}-\mathrm{Si} 3-\mathrm{Gd}^{\mathrm{xix}}$ & $129.93(3)$ \\
\hline $\mathrm{Si} 2^{\mathrm{xiii}}-\mathrm{Re} 2-\mathrm{Re} 2^{\text {viii }}$ & 90.0 & $\mathrm{Si} 3^{\mathrm{xvii}}-\mathrm{Si} 3-\mathrm{Gd}^{\mathrm{xix}}$ & $63.68(8)$ \\
\hline $\mathrm{Si} 2-\operatorname{Re} 2-\operatorname{Re} 2^{\text {viii }}$ & 90.0 & $\mathrm{Gd}^{\mathrm{ii}}-\mathrm{Si} 3-\mathrm{Gd}^{\mathrm{xix}}$ & $127.36(16)$ \\
\hline $\operatorname{Re} 2^{\text {xiv }}-\operatorname{Re} 2-\operatorname{Re} 2^{\text {viii }}$ & 180.0 & $\mathrm{Gd}^{\mathrm{xviii}}-\mathrm{Si} 3-\mathrm{Gd}^{\mathrm{xix}}$ & $78.66(6)$ \\
\hline $\mathrm{Si}^{\mathrm{ii}}-\mathrm{Re} 2-\mathrm{Gd}^{\mathrm{viii}}$ & $168.29(6)$ & $\operatorname{Re} 1-\mathrm{Si} 3-\mathrm{Gd}^{\mathrm{xx}}$ & $129.93(3)$ \\
\hline $\mathrm{Si}^{\mathrm{xii}}-\mathrm{Re} 2-\mathrm{Gd}^{\mathrm{viii}}$ & $59.57(4)$ & $\mathrm{Re}^{\mathrm{x}}-\mathrm{Si} 3-\mathrm{Gd}^{\mathrm{xx}}$ & $81.779(12)$ \\
\hline $\mathrm{Si}^{\mathrm{v}}{ }^{\mathrm{iii}}-\mathrm{Re} 2-\mathrm{Gd}^{\text {viii }}$ & $56.71(8)$ & $\operatorname{Re} 1^{i x}-S i 3-G d^{x x}$ & $151.41(5)$ \\
\hline $\mathrm{Si} 1-\mathrm{Re} 2-\mathrm{Gd}^{\mathrm{viii}}$ & $94.36(7)$ & $\operatorname{Re} 1^{x i}-S i 3-G d^{x x}$ & $67.712(11)$ \\
\hline $\mathrm{Si} 2^{\mathrm{xii}}-\mathrm{Re} 2-\mathrm{Gd}^{\mathrm{viii}}$ & $64.270(10)$ & $\mathrm{Si} 3^{\mathrm{xvii}}-\mathrm{Si} 3-\mathrm{Gd}^{\mathrm{xx}}$ & $63.68(8)$ \\
\hline $\mathrm{Si} 2-\mathrm{Re} 2-\mathrm{Gd}^{\mathrm{viii}}$ & $115.730(10)$ & $\mathrm{Gd}^{\mathrm{ii}}-\mathrm{Si} 3-\mathrm{Gd}^{\mathrm{xx}}$ & $78.66(6)$ \\
\hline $\operatorname{Re} 2^{\text {xiv }}-\operatorname{Re} 2-\mathrm{Gd}^{\text {viii }}$ & $114.889(4)$ & $\mathrm{Gd}^{\mathrm{xviii}}-\mathrm{Si} 3-\mathrm{Gd}^{\mathrm{xx}}$ & $127.36(16)$ \\
\hline $\operatorname{Re} 2^{\text {viii_-Re2- }-G^{\text {viii }}}$ & $65.111(4)$ & $\mathrm{Gd}^{\mathrm{xix}}-\mathrm{Si} 3-\mathrm{Gd}^{\mathrm{xx}}$ & $78.66(6)$ \\
\hline
\end{tabular}

Symmetry codes: (i) $-y+1 / 2,-x+1 / 2, z-1 / 2$; (ii) $-y+1 / 2,-x+1 / 2, z+1 / 2$; (iii) $-x+1 / 2, y+1 / 2,-z+1 / 2$; (iv) $x+1 / 2,-y+1 / 2, z-1 / 2$; (v) $-y+1, x,-z$; (vi) $-y+1$, $x, z$; (vii) $x, y,-z$; (viii) $-x,-y+1,-z$; (ix) $y,-x,-z$; (x) $-x,-y,-z$; (xi) $-y, x, z$; (xii) $y-1 / 2, x+1 / 2,-z+1 / 2$; (xiii) $-x,-y+1, z$; (xiv) $-x,-y+1,-z+1$; (xv) $y$, $-x+1,-z$; (xvi) $x, y,-z+1$; (xvii) $-x,-y,-z+1$; (xviii) $-x+1 / 2, y-1 / 2,-z+1 / 2$; (xix) $y-1 / 2, x-1 / 2,-z+1 / 2$; (xx) $x-1 / 2,-y+1 / 2, z+1 / 2$. 